\title{
ON THE NATURE OF COHERENTS IN THE SYSTEM OF OSCILLATORS
}

\author{
V.M. Kuklin \\ V.N. Karazin Kharkiv National University, Kharkiv, Ukraine \\ E-mail:v.m.kuklin@karazin.ua
}

The paper presents the transition to the regime of induced radiation of a system of oscillators in the classical and the quantum cases. This transition occurs due to synchronization by the integral field of the phases of a small part of oscillator-emitters. In the quantum analogue of this model, it is shown that the formation of an induced (and, therefore, coherent, as noted by Ch. Towns) pulse of the field is due to the interference of nutation of population inversion in different regions of the system of oscillators. The law of spatial variation of the field intensity is determined by the dispersion characteristics of the system and the level of absorption or output of the radiation energy. Only a small fraction of oscillators provide induced radiation: $8 \%$ in the classical case and half as much in the case of a quantum system, where a change in the sign of population inversion in the regions of the highest field values significantly affects the limitation of the radiation intensity.

PACS: 05.45.Xt, 52.40.Mj

\section{INTRODUCTION}

In the famous work [1] R.H. Dicke considered the interaction of oscillators or emitters that are close to each other and believed that they were actually merged into one quasiparticle. Note that in the quantum case it is not about phase synchronization of oscillators, as in the classical consideration, but about an increase in the probability of radiation, which actually leads to the same result. In this case, their wave functions overlap and the probability of spontaneous emission of this quasiparticle increases in comparison with the probability of emission of individual oscillators or emitters. However, if the oscillators or emitters are spaced apart in space, the overlap of their wave functions becomes either weak or imperceptible in general. In most existing lasers, the density of active particles is such that the distances between them are quite significant and one should not expect overlapping of wave functions.

Indicative in this sense is the velocity distribution of free electrons in semiconductors [2], if, of course, the density of these electrons are sufficiently small, for example, at low temperatures. In this case, their distribution is in fact Maxwellian, and their temperature is approximately equal to the temperature of the atomic system. And the thing is that the Fermi distribution is possible only if the wave functions of electrons overlap, which can be observed in metals, where the number of free electrons is comparable with the number of atoms. That is, the distance between free electrons in metals is so small that overlapping of their wave functions occurs.

How do electrons of active atoms interact in lasers? Their interaction is due to electromagnetic radiation fields. In the quantum case, when the phases of the oscillator and the field are not determined, only the relative orientation of the radiating dipole and the electric field is indicated, that is, only the projections of the dipole moment on the direction of the electric field are known, the role of the Rabi frequency can be determining.

Many authors note that the Rabi frequency determines the oscillatory nature of the change in the population inversion of a system of radiating dipoles (nutation), which have ground and excited energy levels and, generally speaking, is proportional to the probability of induced emission and absorption of field quanta [3, 4].
Below it is shown that it is precisely the quasi-periodic changes in population inversion in different areas with the Rabi frequency, which depends on the local field intensity in these areas, lead to an increase in the intensity of the integral field in the system. Also, as in the case of the classical description of an open system of oscillators [5]. In the quantum analogue of this problem one can see that only a small part of the oscillators are involved in the creation of an induced field.

\section{EXAMPLE OF CLASSICAL DESCRIPTION OF RADIATION OF A ONE-DIMENSIONAL SYSTEM OF OSCILLATORS}

In the one-dimensional case, we consider the processes of generation of electromagnetic waves by a system of oscillators with fixed centers [5]. Let the frequency of the wave and the frequency of the oscillators coincide and be equal $\omega$. Wave vector of oscillations $\vec{k}=(0,0, k)$, field components $\vec{E}=(E, 0,0), \vec{B}=(0, E, 0)$ and $E=|E| \cdot \exp \{-i \omega t+i k z+i \varphi\}$. Oscillators are located along the axis $O Z$ in quantity $N$ at the wavelength $2 \pi / k$. The mass of the oscillator is equal $m$, the charge is equal $-e$. The initial oscillation amplitude of the oscillator is equal to $a_{0}$. We assume that the oscillator moves only in the direction of the axis X. In this case, the influence of the magnetic field of the wave on the oscillator dynamics can be neglected.

The equations describing the excitation of a field by an oscillator current in such a one-dimensional representation $j_{x}=-e a \omega_{0} \cdot \operatorname{Cos}\left(\omega_{0} t-\psi\right) \cdot \delta\left(z-z_{0}\right)$ whose coordinates can be written as $\vec{r}=\left(a \cdot \operatorname{Sin}\left(\omega_{0} t-\psi\right), 0, z_{0}\right)$

$$
\begin{aligned}
& \frac{\partial^{2} E_{x}}{\partial z^{2}}-\frac{1}{c^{2}} \frac{\partial^{2} D_{x}}{\partial t^{2}}=\frac{4 \pi}{c^{2}} \frac{\partial J_{x}}{\partial t}= \\
& =\frac{4 \pi}{c^{2}} e a \omega^{2} \operatorname{Sin}(\omega t-\psi) \cdot \delta\left(z-z_{0}\right) .
\end{aligned}
$$

We will seek a solution for the amplitude of the electric field of the wave in the form $E_{x}=E \cdot \exp \left\{-i \omega_{0} t+i k z\right\}$. For the slowly varying in space amplitude of the radiation field, the equation 


$$
\begin{aligned}
& \frac{\partial^{2} E_{x}}{\partial z}=e a \omega^{2} M \exp \left\{i \psi-i k z_{0}\right\} \cdot \delta\left(z-z_{0}\right)= \\
& =\lambda \cdot \delta\left(z-z_{0}\right),
\end{aligned}
$$

whose solution

$$
E=C+\lambda \cdot \theta\left(z-z_{0}\right),
$$

where $C$ is a constant to be defined.

The dispersion equation for emitted radiation by the oscillator is $D(\omega, k) \equiv\left(\omega^{2} \varepsilon_{0}-k^{2}\right)=0$, the roots of which

$$
\begin{aligned}
& k_{1,2}= \pm\left(\omega \operatorname{Re} \varepsilon_{0} / c\right)\left(1+\operatorname{Im} \varepsilon_{0} / \operatorname{Re} \varepsilon_{0}\right) \approx \\
& \approx \pm\left(\omega \varepsilon_{0} / c\right)(1+i 0),
\end{aligned}
$$

for a wave propagating in the direction $z>z_{0}$, the wave number $k=k_{1}>0$ and the value of the constant $C$ should be equal to zero, in order to run away from the unlimited growth of the field at infinity. For a wave propagating in the direction $z<z_{0}$, the wave number is $k=k_{2}<0$, a constant value for the same reasons should be chosen equal $-\lambda$. The amplitude of the electric field while

$$
\begin{aligned}
& E_{x}=\pi e a \omega_{0} M \cdot c^{-1} \exp \{-i \omega t+i \psi\} \times \\
& \times\left[\operatorname { e x p } \left\{i k_{0}\left(z-z_{0}\right) \cdot U\left(z-z_{0}\right)+\right.\right. \\
& +\exp \left\{-i k\left(z-z_{0}\right) \cdot U\left(z-z_{0}\right)\right\},
\end{aligned}
$$

where $U(z)=1$ at $z \geq 0$ and $U(z)=0$ at $z<0$, herewith $M=n_{0} b, n_{\mathrm{C}}$ is the density of particles in a unit of volume, $\boldsymbol{b}$ is the length of the considered space in the longitudinal direction. For one particle in such a volume of a single section and length $b, M$ it is numerically equal to one. You can find the Rabi frequency value for a single dipole system for this case.

$$
\Omega_{1}=4 \pi^{2} e^{2} a^{2} M \omega c^{-1} h^{-1} .
$$

Generally speaking, this is the inverse time of the inversion change (in the absence of relaxation processes due to interaction with the external environment), or in this case, this value may have the meaning of the radiation probability of the excited dipole quantum [3]. Indeed, multiplying the field energy density in the onedimensional case $\left(E_{x}^{2} / 4 \pi\right)$ by the volume occupied by the emitted radiation in two directions over time $\Omega_{1}^{-1}$ (since $M$ is numerically equal to one), we obtain the quantum energy

$$
\left(E_{x}^{2} / 4 \pi\right) c \frac{2}{\Omega_{1}}=M h \omega / 2 \pi .
$$

That is, the reciprocal of the Rabi frequency $\Omega_{1}{ }^{-1}$, in this case, is numerically equal to the time during which one energy quantum is emitted (or, which is the same, the probability of aunpromptedemission of a quantum by a classical oscillator, which corresponds to the sense of the probability of spontaneous emission. Note that the phase of the radiation field and the phase of the oscillator are obviously consistent, for this is its own field.

In the general field of many particles in $z_{j} \in 0 \ldots b$, the equation of motion for a separate oscillator take the form

$$
\left(d^{2} r / d t^{2}+\omega_{0}^{2} r\right)=-\frac{e}{m} E_{x}(z, t) .
$$

Using the expressions below

$$
\begin{gathered}
\mathrm{E}=\frac{e E}{m \omega_{0} a_{0} \gamma}, \frac{\pi e^{2} M}{2 m c}=\gamma ; A_{s}=\frac{k_{0} a_{s}}{k_{0} a_{0}}=a_{s} / a_{0} ; \\
M=n_{0} b ; 2 \pi Z=k_{0} z, \tau=\gamma t ; \\
\mathrm{E}_{0}=\frac{e E_{0}}{m \omega_{0} \gamma a_{0}}, \frac{2 c}{b \gamma_{0}}=\theta \quad \alpha=\frac{3 \omega_{0}}{4}\left(k_{0} a_{0}\right)^{2},
\end{gathered}
$$

we present a system of equations describing the process of forming an integral field in a system of oscillators in a dimensionless form [6]

$$
\begin{aligned}
& \frac{d}{d \tau} A_{j}=\mathrm{E}_{0} \operatorname{Cos}\left\{2 \pi Z_{j}-\psi_{j}\right\}-(N)^{-1} \sum_{s=1}^{N} A_{s} \times \\
& \times\left[\operatorname{Cos}\left\{-\psi_{j}+2 \pi\left(Z_{j}-Z_{s}\right)+\psi_{s}\right\} U\left(Z_{j}-Z_{s}\right)+\right. \\
& \left.\operatorname{Cos}\left\{-\psi_{j}-2 \pi\left(Z_{j}-Z_{s}\right)+\psi_{s}\right\} U\left(Z_{s}-Z_{j}\right)\right], \\
& A_{j}\left[\frac{d \psi_{j}}{d \tau}-\alpha\left(A_{j}^{2}-A_{j 0}^{2}\right)\right]=\mathrm{E}_{0} \operatorname{Sin}\left\{2 \pi Z_{j}-\psi_{j}\right\}- \\
& -(N)^{-1} \sum_{s=1}^{N} A_{s} \times \\
& \times\left[\operatorname{Sin}\left\{-\psi_{j}+2 \pi\left(Z_{j}-Z_{s}\right)+\psi_{s}\right\} U\left(Z_{j}-Z_{s}\right)+\right. \\
& \left.+\operatorname{Sin}\left\{-\psi_{j}-2 \pi\left(Z_{j}-Z_{s}\right)+\psi_{s}\right\} U\left(Z_{s}-Z_{j}\right)\right],
\end{aligned}
$$

where $\theta=\delta / \gamma_{0}>1, \gamma_{0}^{2}=\pi e^{2} M / 2 m c$, and $\gamma=\gamma_{0}^{2} / \delta$, the term proportional $\alpha$ takes into account the weak relativism of the oscillator mass. By the way, in the theory of cyclotron generators such nonlinearity is a consequence of the so-called negative mass effect. Accounting for such nonlinearity may be significant, since in [7] noted, that in the system of linear oscillators the generation efficiency is insignificant.

The field slowly varying in time, generated by oscillators inside and outside the area occupied by them, has the form

$$
\begin{aligned}
& \mathrm{E}(Z, \tau)=\frac{1}{N} \sum_{s=1}^{N} A_{s} \exp \left\{i \psi_{s}\right\}\left[\exp \left\{2 \pi i\left(Z-Z_{s}\right)\right\} \times\right. \\
& \left.\times U\left(Z-Z_{s}\right)+\exp \left\{-2 \pi i\left(Z-Z_{s}\right)\right\} U\left(Z_{s}-Z\right)\right],
\end{aligned}
$$

that is, expression (10) is a slowly varying envelope of the HF field oscillations. Note that due to the removal of energy from a small active zone, the field does not accumulate in its volume.

For the number of oscillators $N=10^{4}$, the average integral field $|\mathrm{E}|$ of a system of oscillators with a random distribution of the initial phase (which in the classical case can be considered spontaneous) is 100 times smaller than the maximum possible field value in the case when all oscillators are phase-locked. That is, for fields of spontaneous and absolutely coherent induced radiation, the relation is satisfied $10^{-2}: 1$. For squares of amplitudes $|\mathrm{E}|^{2}$, this ratio takes the form $10^{-4}: 1$. Below we will discuss the results of solving the system (8), (9), carried out previously with D. Litvinov [5].

The greatest value of the field in the opposite direction of the propagation of the external wave side of the system $|\mathrm{E}| \approx 0.23 \ldots 0.25$ and $|\mathrm{E}|^{2} \approx 0.08$. The external stabilizing field is small $\mathrm{E}_{0}=0.05$ at the same time. And since in the case of fully phased emitters $|\mathrm{E}|^{2}=1$, the degree of coherence in this case is $8 \%$. Numerical analysis of system (8) - (10) showed that a significant ISSN 1562-6016. BAHT. 2019. №4(122) 
number of particles practically do not participate in the generation of a coherent field (Figs. 1, 2).

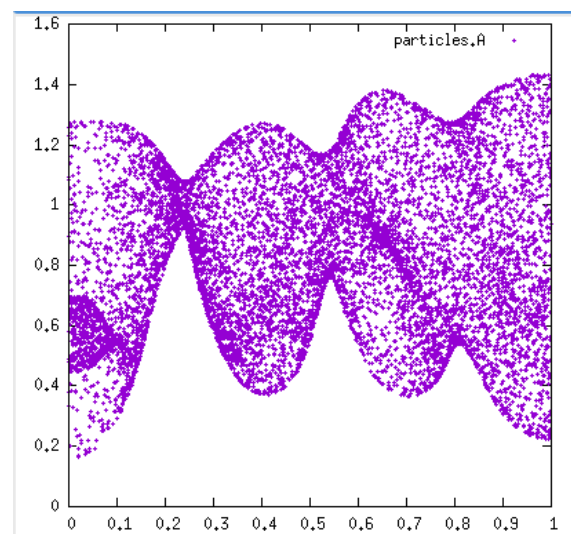

Fig. 1. The amplitude of oscillators at different points along $Z$ at the moment, when the field reaches a maximum $(\Delta Z=1, \alpha=1)$ [5]

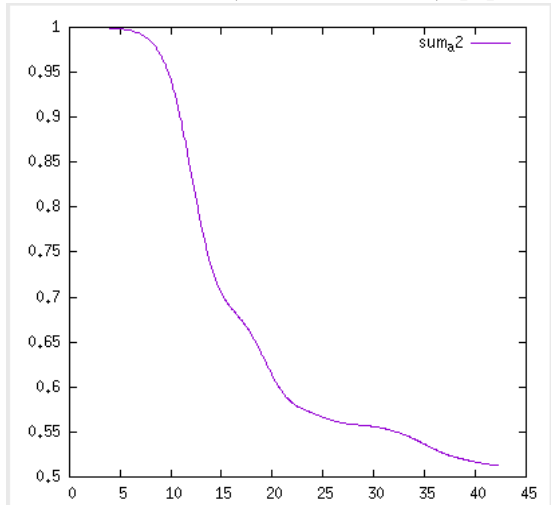

Fig. 2. The normalized total energy of the particles as a function of time. $\Delta Z=1, \alpha=1$ [5]

Although the coherence level of $8 \%$ is quite significant, because the intensity of this field is proportional $0.08 \cdot \mathrm{N}^{2}$, which is 800 times greater than the intensity of spontaneous emission, understood in the classical analogue of superradiance considered here as the sum of the intensities of individual uncorrelated emitters. Obviously, with a larger number of particles, this difference between superradiance and spontaneous emission of particles with random phases will only increase.

\section{SEMICLASSICAL MODEL OF RADIATION}

If the quantum system is in the waveguide medium, then the semiclassical theory should be used to describe the generation processes, in particular, used previously in the works of Yu.L. Klimontovich and colleagues [8, 9]. The system of one-dimensional equations of the semi classical theory for the amplitudes of the perturbations of the electric field, polarization, describing the excitation of electromagnetic oscillations in a two-level active medium represent as follows:

$$
\begin{gathered}
\frac{\partial^{2} E}{\partial t^{2}}+\delta \frac{\partial E}{\partial t}-c^{2} \frac{\partial^{2} E}{\partial x^{2}}=-4 \pi \frac{\partial^{2} P}{\partial t^{2}}, \\
\frac{\partial^{2} P}{\partial t^{2}}+\omega^{2} P=-\frac{4 \pi \omega\left|d_{a b}\right|^{2}}{h} \mu E,
\end{gathered}
$$

to which you need to add an equation for population inversion that slowly changes with time

$$
\frac{\partial \mu}{\partial t}=\frac{4 \pi}{h \omega}<E \frac{\partial P}{\partial t}>.
$$

We assume that the frequency of the transition between energy levelscorresponds to the field frequency, the line width in the equation for polarization and the inversion relaxation due to external causes are neglected.

Let be $\delta$ - the decrement of field absorption in the medium, $d_{a b}$ - the matrix element of the dipole moment (or rather its projection on the electric field direction), the difference populations $\mu=n \cdot\left(\rho_{a}-\rho_{b}\right)$ per unit volume, $\rho_{a}$ and $\rho_{b}$ relative populations of levels in the absence of a field. Fields are represented by $E=[E(t) \exp \{-i \omega t\}+E \times(t) \exp \{i \omega t\}] / 2 \quad$ and $P=[P(t) \exp \{-i \omega t\}+P \times(t) \exp \{i \omega t\}] / 2, \quad$ and $<E^{2}>=|E(t)|^{2} / 2$.

For slowly varying field amplitudes and polarizations, the following equations are valid:

$$
\begin{gathered}
\frac{\partial E(t)}{\partial t}+\delta \cdot E(t)+v_{g} \frac{\partial E(t)}{\partial x}=2 i \pi \omega P(t), \\
\frac{\partial P(t)}{\partial t}=\left|d_{a b}\right|^{2}(2 \pi \mu / h) \frac{E(t)}{i} .
\end{gathered}
$$

In the homogeneous case, you can get the equation

$$
\frac{\partial^{2} E(t)}{\partial t^{2}}+\delta \frac{\partial E(t)}{\partial t}=\frac{4 \pi^{2} \omega\left|d_{a b}\right|^{2}}{h} \mu E(t) .
$$

From which it follows that the field in the absence of losses can grow with increment

$$
\gamma=\left(\frac{4 \pi^{2} \omega\left|d_{a b}\right|^{2}}{h} \mu\right)^{1 / 2}
$$

Since in this case, (13) can be rewritten in the form

$$
\frac{d \mu}{d t}+\sigma=-\left(\frac{d}{d t}+2 \delta\right) N_{k},
$$

where in $\sigma$ we take into account the inversion losses associated with spontaneous radiation, $N_{k}=|E|^{2} / 2 h \omega$ is the number of field quanta per unit volume.

Taking into account the field energy loss in the system without active particles with a decrement equal $\delta$ to when $\delta>\gamma$ leads to the fact that equations (14) and (15) imply the relation

$$
\begin{aligned}
& \delta \frac{\partial N_{k}}{\partial t}=\frac{8 \pi^{2} \omega\left|d_{a b}\right|^{2} \mu}{h} N_{k}= \\
& =4 \pi^{2} \frac{\left|d_{a b}\right|^{2}|E|^{2}}{h^{2}} \mu=\frac{\Omega_{N}^{2}}{2} \mu .
\end{aligned}
$$

This case corresponds to the classical analogue considered above with $\delta=2 v_{g} / b$, where $b$ is the longitudinal size of the system. We will first interest in the behavior of population inversion. For sufficiently large losses of field energy in the medium $\delta>\gamma$, from equations (18) and (19) we get

$$
\frac{\partial^{2} \mu}{\partial t^{2}}+\Omega_{N}^{2} \mu=0
$$

where $\Omega_{N}=\left[\frac{8 \pi^{2}\left|d_{a b}\right|^{2}}{h^{2}}|E|^{2}\right]^{1 / 2}$ is the Rabi frequency, which has the meaning of the inverse time of the change 
in the inversion and the probability of the induced radiation under the influence of the field [3,4]. It is important to note that the Rabi frequency increases in proportion to the magnitude of the increasing electric field described by equation (19), that is, the rate of change of the inversion is accelerated. Consequently, the increase in the probability of radiation of excited dipoles at each point in space in the semiclassical description occurs under the influence of the integral electric field. On the other hand, the energy output from the system leads to a decrease in the initial integral level of population inversion. Let us discuss this phenomenon in the simplest model used above for the classical description of the system of oscillators above.

It is of interest to find out the nature of the change in population inversion in space. Consider a limited unit volume with a longitudinal size equal to the length of the electromagnetic wave, assuming that the configuration of the field intensity distribution does not change (since it is determined only by the properties of the waveguide system and the nature of the energy output from it). We use the following expression $\tau=\gamma t=t\left(\frac{4 \pi \omega\left|d_{a b}\right|^{2}}{\hbar} \mu_{0}\right)^{1 / 2}, \frac{N_{k}(t)}{\mu_{0}}=N$, and the initial value of the relative number of quanta is small $N(0)=0.001$. The distribution of the field in a unit volume will be presented for each spatial interval in the longitudinal direction in the form $N_{j}=2 \cdot N \cdot \operatorname{Cos}^{2}\left\{2 \pi \frac{j}{S}\right\}$, where $0<j \leq S$, so that the sum $2 \operatorname{Cos}^{2}\left\{2 \pi \frac{j}{S}\right\}$ of all $j$ is equal to unit. In each interval $j$, the value of the initial relative value of the population inversion $M_{j}(0)=\frac{\mu_{j}(0)}{\mu_{0}}=\frac{1}{S}$, where $\sum_{j=1}^{S} \mu_{j}(0)=\mu_{0}$ is the value of the inversion in the whole volume. Equation (20) can be written for each spatial interval in the following form

$$
\frac{d^{2} M_{j}}{d \tau^{2}}=-N_{j} M_{j}=-2 N \operatorname{Cos}^{2}\left\{2 \pi \frac{j}{S}\right\} M_{j} .
$$

For the field, you can, using (18), write the equation

$$
N=-\frac{1}{\theta} \frac{d}{d \tau} \sum_{j=1}^{S} M_{j}-\Sigma
$$

where $\theta=\delta / \gamma>1, \Sigma=\sigma /\left(\delta \cdot \mu_{0}\right)$.

The solution of the system of equations (21), (22) is shown in Figs. 3-6 with the number of layers $S=100$, $\theta=3$ is the level of energy output, the initial conditions $\Sigma=N(0)=0,0001, \mathrm{M}_{j}(0)=1 / S, Z_{j}(0)=-6 \cdot 10^{-6}$, $\Delta \tau=0,05$ is the calculation step, where $Z_{j}(0)=-\theta[\Sigma+N(0)] / S$.

The inversion values $M_{j}$ in each segment of the system are oscillatory damped (due to output or energy loss). This character is similar to the known optical nutation of an open two-level system.

The greater the field intensity in the segment, the greater the frequency of population inversion oscillations (Rabi frequency) in this local region. The interference of these oscillations in different spatial regions (segments) of the system leads to changes in the integral field. In Fig. 3 shows the population inversion distribu- tion along the system at a time instant, in particular, corresponding to the maximum field amplitude (see Fig. 4).

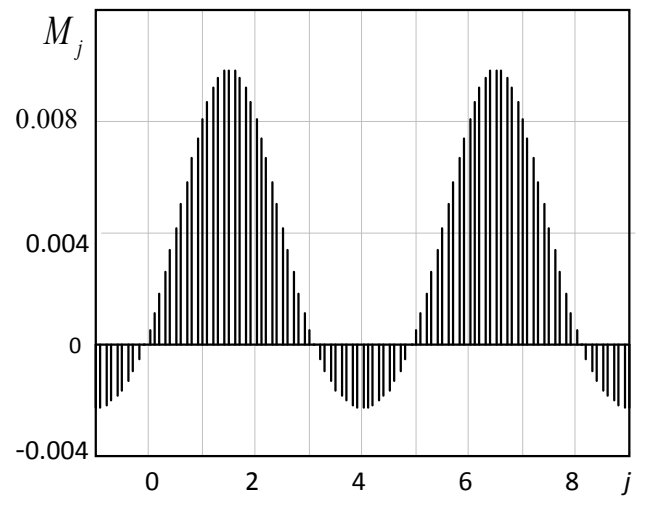

Fig. 3. Inversion levels $M_{j}$ along the system at the moment of maximum field $(t=22.1)$

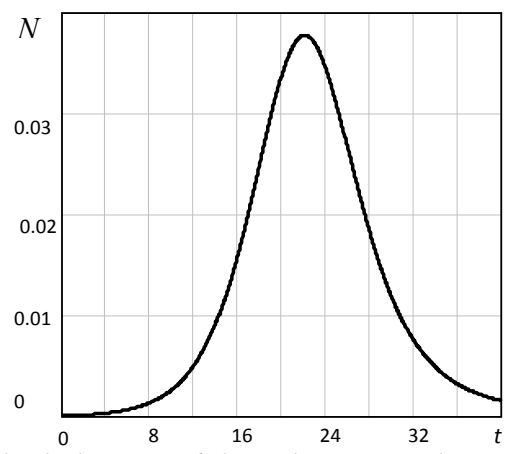

Fig. 4. The behavior of the relative number of quanta

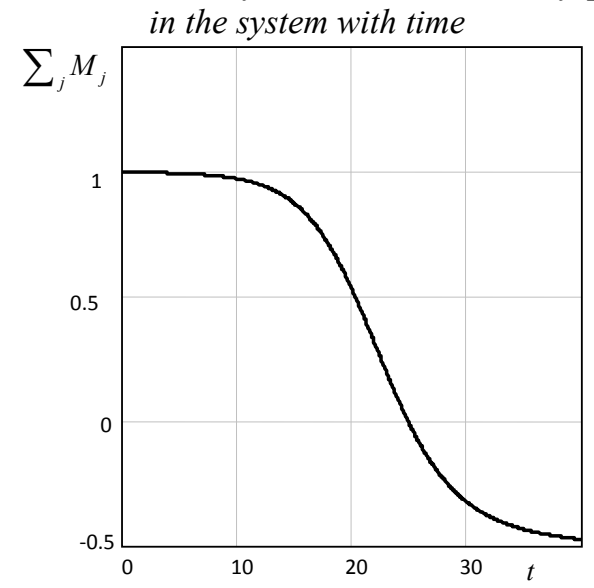

Fig. 5. The behavior of the integral value of inversion

$$
\text { in system } \sum_{j=1}^{S} M_{j}
$$

Attention should be paid to negative values of inversion in the areas of greatest field intensity. Note that in these areas, the processes of induced absorption of the field energy dominate. The negative values of inversion in the areas of greatest field intensity limit the growth of the field in the system.

A decrease of the inversion in these areas occurs both due to the nutation of the local inversion (see Fig. 6), and due to absorption (radiation from the system) of the field energy. As in the classical case discussed above, the total energy supply in the system (here, the integral inversion of populations) decreases (see Fig. 5 and compare with Fig. 2). In this case, less than $4 \%$ of atoms contribute to the induced radiation at the maximum of the field pulse, which is almost two times less than in the classical analogue discussed above. 


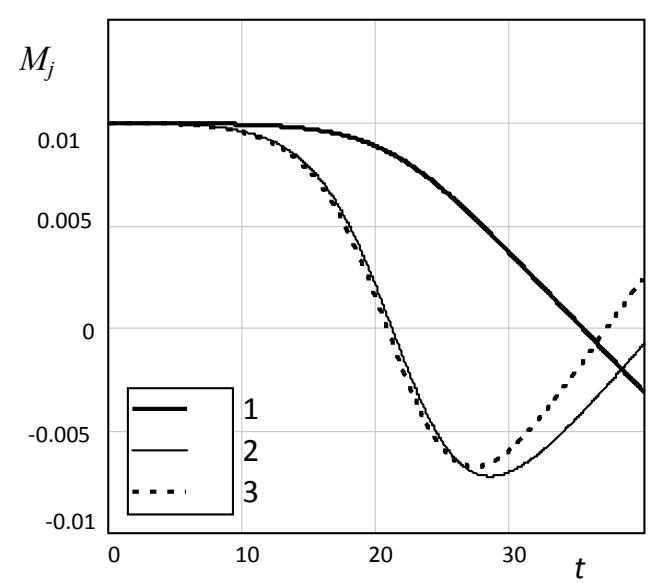

Fig. 6. The change in inversion in different parts of the system with time: $1-M_{30} ; 2-M_{45} ; 3-M_{50}$

\section{CONCLUSIONS}

Thus, in the classical case, the transition to the induced mode and, as noted by C. Towns, is largely coherent radiation [10], Occurs due to synchronization by the integrated field of the phases of a part of oscillatoremitters. In the quantum case, the formation of an induced field pulse occurs due to the interference of nutation of population inversion in different regions of space of the oscillator system (where the dispersion characteristics of the system and the level of absorption or output of the radiation energy determine the law of spatial variation of the field intensity).

As the population inversion decreases, the number of radiated field quanta increases. If the inversion increases, the induced absorption of quanta by a twolevel system increases accordingly. On the other hand, the absorption or output of the radiation energy leads to a decrease in the field intensity in the active zone. The competition of all these processes leads to the fact that the Rabi frequency (as well as the probability of induced radiation with a positive inversion or induced absorption with its negative value) changes, and nonuniformly over the space of the active zone. These processes form a pulse of coherent radiation. We note a small fraction of oscillators that provide induced radiation: $8 \%$ in the classical case and half as much in the case of a quantum system. The change in the sign of population inversion in the regions of the highest field values in the system significantly affects the limitation of the radiation intensity.

In conclusion, I consider it my duty to express my gratitude to D.N. Litvinov and E.V. Poklonsky for assistance in numerical calculations, V.A. Buts, V.I. Karas' and V.V. Yanovsky for discussing the materials of the work.

\section{REFERENCES}

1. R.H. Dicke. Coherence in spontaneous radiation processes // Phys. Rev. 1954, v. 93, p. 99.

2. F.G. Bass, Yu.G. Gurevich. Hot electrons and strong electromagnetic waves in semiconductor plasma and gas discharge. M.: "Science", 1975, $400 \mathrm{p}$.

3. A.S. Davydov. Quantum mechanics. M.: "Fizmatgiz", 1963, $748 \mathrm{p}$.

4. L. Allen, J. Eberly. Optical rfsonance and two-level atoms // Witey-Interscicitce Publication John Witty and Sons. New York-London-Sydney-Toronto. 1975, $222 \mathrm{p}$.

5. V.M. Kuklin. Selected chapters (theoretical physics). Kh.: "KhNU". 2018, $224 \mathrm{p}$

6. V.M. Kuklin, D.N. Litvinov, V.E. Sporov. Super radiance of stationary oscillators // Problem of Atomic Science and Technology. Series "Plasma Electronics and New Methods of Acceleration”. 2018, № 4, p. 217-220.

7. Yu.A. Il'inskii, N.S. Maslova. Classical analogue of super radiance in a system of interacting nonlinear oscillators // Zh. Exp. Teor. Fiz. 1988, v. 91, № 1, p. 171-174.

8. S.G. Zeiger, Yu.L. Klimontovich, P.S. Landa, E.G. Lariontsev, E.E. Fradkin. Wave and fluctuation processes in lasers. M.: "Science", 1974.

9. P.S. Landa. Auto-oscillations in distributed systems. M.: "Science", 1983.

10. Ch. Towns. Obtaining coherent radiation with the help of atoms and molecules // UFN. 1966, v. 88, № 3, p. 461-483.

Article received 14.05.2019

\section{О ПРИРОДЕ КОГЕРЕНТНОСТИ В СИСТЕМЕ ОСЦИЛЛЯТОРОВ}

\section{В.М. Куклин}

Представлен переход в режим индуцированного излучения подобных систем осцилляторов в классическом и квантовом случаях. Этот переход происходит из-за синхронизации интегральным полем фаз небольшой части осцилляторовизлучателей. В квантовом аналоге этой модели показано, что формирование индуцированного (а следовательно, когерентного, как отмечал еще Ч. Таундс) импульса поля происходит из-за интерференции нутаций инверсии населенностей в разных областях пространства системы осцилляторов, где закон пространственного изменения интенсивности поля определяется дисперсионными характеристиками системы и уровнем поглощения или вывода энергии излучения. Лишь небольшая доля осцилляторов обеспечивает индуцированное излучение: $8 \%$ в классическом случае и вдвое меньше в случае квантовой системы, где на ограничение интенсивности излучения существенно влияет изменение знака инверсии населенностей в областях наибольших значений поля в системе.

\section{ПРО ПРИРОДУ КОГЕРЕНТНОСТІ В СИСТЕМІ ОСЦИЛЯТОРІВ}

\section{В.М. Куклін}

Представлено перехід у режим індукованого випромінювання подібних систем осциляторів у класичному і квантовому випадках. Цей перехід відбувається через синхронізації інтегральним полем фаз невеликої частини осциляторіввипромінювачів. У квантовому аналозі цієї моделі показано, що формування індукованого (а отже, когерентного, як відзначав ще Ч. Таундс) імпульсу поля відбувається через інтерференцію нутації інверсії заселеності в різних областях простору системи осциляторів, де закон просторової зміни інтенсивності поля визначається дисперсійними характеристиками системи і рівнем поглинання або виведення енергії випромінювання. Лише невелика частка осциляторів забезпечує вимушене випромінювання: 8\% у класичному випадку і вдвічі менше в разі квантової системи, де на обмеження інтенсивності випромінювання істотно впливає зміна знаку інверсії заселеності в областях максимальних значень поля в системі. 